\title{
Vascular cognitive impairment
}

\author{
Dragos Catalin Jianu ${ }^{1,2}$, Claudia Barsan² \\ ${ }^{1}$ Department of Neurology, "Victor Babes" University of Medicine and Pharmacy, Timisoara, Romania \\ ${ }^{2}$ First Department of Neurology, Clinical Emergency County Hospital, Timisoara, Romania
}

\begin{abstract}
Vascular cognitive impairment $(\mathrm{VCl})$ is a heterogeneous group of cognitive disorders that share a presumed vascular cause and it is the second most common cause of cognitive impairment in the elderly after Alzheimer's disease. Its prevalence is estimated to double over the next 30 years. Vascular cognitive impairment encompasses cases related to multiple cortico-subcortical infarcts, silent infarcts, strategic infarcts, small vessel disease, as well as mixed pathology. It is important to emphasize that some of the risk factors for vascular dementia are the same as for stroke, including high blood pressure, diabetes, heart disease and cigarette smoking. Compared with Alzheimer's disease, vascular dementia has a higher rate of mortality and a slower rate of cognitive decline. The aim of the treatment in vascular cognitive impairment is mainly to identify and reduce the effect of the vascular risk factors. This article aims to resume the most important data regarding $\mathrm{VCl}$.
\end{abstract}

Keywords: cognitive impairment (VCI), dementia, risk factors, cerebrovascular disease

\section{INTRODUCTION}

Vascular cognitive impairment (VCI) is a heterogeneous group of cognitive disorders that share a presumed vascular cause $(1,2)$. It includes the whole spectrum of cognitive alterations greater than expected for normal aging, attributed to ischemic or hemorrhagic cerebral insults $(3,4)$. Thus, VCI covers subjects who have cognitive impairment associated with stroke, multiple cortical infarcts, multiple subcortical infarcts, silent infarcts, strategic infarcts, small-vessel disease with white-matter lesions and lacunae (5). VCI cases that do not meet the criteria for dementia can be labeled as VCI with no dementia, vascular cognitive impairment no dementia (vascular CIND) or vascular mild cognitive impairment. This concept also includes dementias where both cardiovascular disease (CVD) and Alzheimer's disease (AD) processes are involved (1). It is important to mention that, unlike AD, VCI is not itself a disease - it oc- curs when vascular risk factors lead to CVD and afterwards to vascular brain injury (VBI) that disrupts brain networks for memory and thinking (4).

Mild cognitive impairment (MCI) is a syndrome characterized by a cognitive decline greater than expected for an individual's age and education level, but with no significant daily functional disability (6-8). Within the MCI concept we distinguish amnestic MCI (a-MCI) from multiple-domain MCI (md-MCI). Amnestic MCI (a-MCI) is defined by a memory deficit in comparison with the patients of a similar age and education level, while md- MCI is characterized by impairment in several cognitive domains (eg, language, executive function, visuospatial skills). Individuals with md-MCI may also have memory impairment. A third subtype of MCI is characterized by a single cognitive domain - impairment without memory involvement $(2,9)$. MCI is regarded as a transitional, reversible stage between normal aging and dementia (10). 
Vascular dementia $(\mathrm{VaD})$ is now considered to be the extreme end of a spectrum of syndromes of cognitive impairment associated with different types of CVD $(1,3)$.

\section{Epidemiology and risk factors}

It is estimated that dementia affects around $5-8 \%$ of those aged 65 years and older, and $30 \%$ of people older than $80(1,5,11)$. VaD is the second most common cause of cognitive impairment in the elderly after $\mathrm{AD}$, constituting $15-20 \%$ of all dementia worldwide $(12,13)$. The incidence of $\mathrm{VaD}$ increases with age, without any significant difference between men and women (1). The Canadian Study of Health and Ageing prospective cohort study of 10,253 elderly persons reported that patients with VCI had a higher rate of institutionalization and death, while half of those who had VCIND developed dementia within 5 years $(13,14,15)$. It is also important to emphasize that stroke prevalence is 6 times higher in those with cognitive impairment relative to normal (16). Also, dementia is observed within 3 months post-stroke with a prevalence as high as $25-33 \%$ and about two-thirds of these patients meet the criteria of $\operatorname{VaD}(3,5)$.

According to a recent study, it is estimated that the prevalence of VCI will double over the next 30 years, making disorders of cognition a priority for healthcare and social-care services $(5,17)$.

It was established that vascular risk factors (VRFs) are associated with cognitive impairment as well as with stroke and AD pathogenesis (18).

The risk factors in dementia are presented in Table 1.

TABLE 1. Dementia risk factors $(1,11,19-24)$

\begin{tabular}{|c|c|c|}
\hline Risk factor & & Observations \\
\hline Vascular disorders & $\begin{array}{l}\text { Cerebrovascular disease: } \\
\text { - clinical stroke } \\
\text { - large-artery atherosclerosis } \\
\text { - cerebral emboli, } \\
\text { - silent brain infarct, } \\
\text { - increased ventricular volume, } \\
\text { - white matter lesions } \\
\text { - cerebral amyloid angiopathy } \\
\text { Cardiovascular disease } \\
\text { - arterial hypertension } \\
\text { - atrial fibrillation, } \\
\text { - myocardial infarction, } \\
\text { - heart failure } \\
\text { Diabetes mellitus } \\
\text { Hyperlipidemia } \\
\text { Overweight and obesity } \\
\text { Systemic inflammation }\end{array}$ & $\begin{array}{l}\text { Usually left hemisphere strokes, anterior and posterior } \\
\text { cerebral arteries territories infarction } \\
\text { High BP is seen as a dementia risk factor independent of the } \\
\text { CVD presence } \\
\text { Diabetes mellitus is considered to be the strongest risk factor } \\
\text { Several prospective reports indicate that overweight (BMI } \\
25-29.99 \text { kg } \mathrm{m}^{2} \text { ) and/or obesity (BMI > } 30 \text { kg } \mathrm{m}^{2} \text { ) increase } \\
\text { dementia risk over long periods of follow-up) (19-22) }\end{array}$ \\
\hline $\begin{array}{l}\text { Lifestyles and } \\
\text { habits }\end{array}$ & $\begin{array}{l}\text { Cigarette smoking } \\
\text { Alcohol consumption } \\
\text { Reduced physical exercise } \\
\text { Dietary/nutritional factors: fats, fish, } \\
\text { polyunsaturated fatty acids, antioxidants, vitamin } \\
\text { B12, folic acid, and high serum homocysteine }\end{array}$ & $\begin{array}{l}\text { Various studies found significant correlations between } \\
\text { smoking and cognitive function }(11,23,24)\end{array}$ \\
\hline $\begin{array}{l}\text { Clustering of } \\
\text { vascular risk } \\
\text { factors }\end{array}$ & $\begin{array}{l}\text { The metabolic syndrome } \\
\text { Vascular risk factor profile: }\end{array}$ & $\begin{array}{l}\text { Referring to aggregation of multiple vascular risk factors } \\
\text { (hypertension, diabetes, heart disease etc). }\end{array}$ \\
\hline $\begin{array}{l}\text { Demographic } \\
\text { factors }\end{array}$ & $\begin{array}{l}\text { Higher age } \\
\text { Low education }\end{array}$ & \\
\hline Genetic factors & Family history and specific genetic features & $\begin{array}{l}\text { The association between } \mathrm{VaD} \text { and } \mathrm{APOE} 4 \text { allele- a well- } \\
\text { established genetic risk factor for } \mathrm{AD} \text {, is debatable and should } \\
\text { be interpreted with caution. }\end{array}$ \\
\hline
\end{tabular}




\section{Neuropathology of $\mathrm{VCl}$}

VCI neuropathology is heterogeneous, thus in the vast majority of cases, the cerebrovascular pathology is associated with other pathologies, usually Alzheimer type $(2,25)$.

Cerebrovascular (CVDs) diseases are the main causes of vascular dementia and an important factor in the development of AD and mixed dementia. (1) According of Riley and colleagues, even in the absence of macroscopic infarctions, patients diagnosed with severe atherosclerotic disease in the circle of Willis have a substantially greater risk (relative risk $>3)$ to develop MCI $(26,27)$.

Cerebrovascular syndromes associated with cognitive decline include:

- multiple cortical, subcortical and silent infarcts or some combination of these;

- single infarcts in strategic regions of the brain (eg: lacunar infarcts affecting the thalamus and basal ganglia that have important interconnections with multiple other cortical parts.

- hemorrhage (intraparechymal and subarachnoid hemorrhage, subdural hematoma)

- hypotension episodes responsible for border-zone infarcts.

- cerebral vasculitis (12).

CVD can be divided into large and small-vessel domains. Large ischemic lesions can be caused by emboli from cardiac origin or from large-vessel disease (LAD) (atherosclerosis, plaque rupture, thrombotic occlusion and dissection), but can also result from haemodynamic events, leading to borderzone lesions. Changes in the small vessels and their subsequent parenchymal lesions are collectively referred to as cerebral small vessel disease (SVD). Abnormalities in these small vessels comprise arteriolosclerosis, lipohyalinosis, fibrinoid necrosis, microatheromas, and cerebral amyloid angiopathy (CAA). Parenchymal lesions secondary to these vessel anomalies include lacunes, diffuse white matter changes and microinfarcts (25).

Another process possibly involved in the pathogenesis of dementia is the chronic microglial activation caused by hypoxia which follow the vascular lesions. The activated microglia produce a series of proinflammatory cytokines, including interleukin (IL)-6, interferon (IFN)- $\gamma$, tumor necrosis factor (TNF)- $\alpha$ and IL-1 $\beta$, through which the inflam- matory response spreads inducing neuronal damage. Supporting these hypothesis, it was reported that patients with vascular dementia showed a high level of serum IL-6 proving the inflammatory component in the development of VCI (28).

\section{$\mathrm{VCl}$ subtypes}

For clinical use, VCI is subdivided according to risk factors, mechanisms, pathology, clinical features, or response to treatment. Thus, VCI encompasses cases related to multiple corticosubcortical infarcts, silent infarcts, strategic infarcts, small vessel disease, as well as mixed pathology (CVD and AD). VCI also include the residual cognitive impairment found in those patients who survive intracerebral and other intracranial hemorrhages $(1,2)$.

The VaD may be divided into the following subtypes:

Cortical VaD (MID or post-stroke VaD). Poststroke dementia develops in up to a third of patients within a year (usually in the first 3 months) after a stroke and is strongly associated with advancing age $(1,2,5)$. It is important to emphasise that a history of stroke rises the risk of subsequent dementia by a factor of $5(1,29,30)$; likewise, a pre-stroke cognitive impairment seems to be a strong predictor of incident of post-stroke dementia, suggesting that $\mathrm{AD}$ may play an etiologic role in $\operatorname{VaD}(1,4)$. Cortical VaD is characterized by a relatively abrupt onset, a step-wise deterioration and a fluctuating course of cognitive functions. Clinically, these patients have, apart from the cognitive impairment, significant focal neurologic signs, consequences of cortical and cortico-subcortical infarcts caused by multiple atherothrombotic and embolic events $(1,16)$.

Subcortical ischemic vascular disease (SIVD) and dementia (small vessel dementia) - is one of the main subtypes of the VaD syndrome. Clinically, it is characterized by the subcortical cognitive syndrome associated with motor hemiparesis, bulbar signs, dysarthria, gait disturbance, depressive illness, emotional lability and deficits in executive functioning. The cognitive deficit is defined by a dysexecutive syndrome (impairment of selective attention, abstract reasoning and mental flexibility), mild memory deficit and behavioral symptoms. The pathological features of SIVD include 
small-vessel degeneration, lacunes, multiple subcortical microinfarcts, focal and diffuse white matter lesions. SIVD encompasses two entities: "the lacunar state" and "Binswanger's disease" $(1,4)$.

Lacunar infarcts represent approximately 25\% of symptomatic ischemic strokes. The lacunae are seen in the cortical white matter or in the corona radiata, internal capsule, centrum semi-ovale, thalamus, basal ganglia or pons $(2,4,5)$.

Binswanger's disease is clinically characterized by the triad: slowly progressive cognitive decline, gait apraxia and early urinary incontinence. Pathological examination of Binswanger's disease cases revealed diffuse or patchy rarefaction of myelin associated with gliosis and spongiosis. The white matter changes described in these patients are consequences of chronic hypoperfusion, incomplete infarction and demyelination in periventricular and occipital deep white border regions $(3,4)$.

Strategic infarct dementia is characterized by focal, frequently small, ischemic lesions involving strategical regions, critical for higher cortical functions. The cortical sites include the hippocampus, angular gyrus and cingulate gyrus, while the subcortical sites are the thalamus, fornix, basal forebrain, caudate, globus pallidus and the genu or anterior limb of the internal capsule $(1,2,31)$. Such strokes can lead to a dementia syndrome by disrupting frontal-subcortical loops $(2,4)$. The amnesia caused by these types of stroke can be distinguished from the episodic memory loss associated with AD dementia disease by the sudden onset of the impairment (16).

Hemorrhagic dementia. Intracerebral and subarachnoid hemorrhages usually present primarily as major strokes with important focal neurologic signs overshadowing the cognitive impairment. Thus, their inclusion or exclusion as classical causes of vascular dementia is debatable $(3,31)$.

Hypoperfusion secondary to carotid diseases is a very rare cause of $\mathrm{VaD}$. Unilateral or bilateral occlusion of carotid arteries usually represent the precipitating factor for dementia. In most cases, pathological examination shows widespread or multifocal cerebral infarction. Chronic ischemia without infarction in the carotid territory is an extremely rare cause of $\mathrm{VaD}$. This type of $\mathrm{VaD}$ is reversible if the hemodynamic deficit disappears (3).

Hereditary vascular dementia (CADASIL). Cerebral autosomal dominant arteriopathy with subcortical infarcts and leukoencephalopathy (CADASIL) is a genetic cerebral small-vessel disease caused by mutations in the NOTCH 3 gene on chromosome 19 (16,32). CADASIL is associated with progressive degeneration of vascular smooth muscle cells which is replaced by granular eosinophilic non-amyloid deposits in the vascular basal lamina $(3,4)$. Clinical manifestations include transient ischemic attacks and strokes (80\%), cognitive deficits $(50 \%)$, recurrent attacks of migraine $(40 \%)$, psychiatric disorders (30\%), and epilepsy (10\%) $(4,5)$. Magnetic Resonance Imaging (MRI) reveals a combination of small lacunar lesions microhemorrhages and diffuse white-matter abnormalities particularly in subcortical regions, typically involving the anterior temporal pole, claustrum and corpus callosum. The diagnosis can be confirmed through genetic tests or skin biopsy, however currently, only symptomatic treatment is available $(4,5,16)$.

\section{DIAGNOSIS}

Different criteria have been proposed for the definition of VaD:

- DSM-IV criteria - sensitive but poorly specific.

- CD-10 criteria - similar to DSM-IV criteria; require the evidence of focal brain damage.

- ADDTC criteria - did not emphasize the presence of specific cognitive domain deficits.

- Hachinski ischemic score - initially developed to differentiate multi-infarct dementia secondary to the accumulation of ischemic lesions (score $4>$ ) from Alzheimer's disease (score $<4)$.

- NINDS-AIREN criteria - currently used for research and clinical trials; incorporate different levels of certainty of the clinical diagnosis (probable, possible, definite); require 1) demonstration of the presence of cognitive impairment, 2) demonstration of the presence of cerebrovascular disease, and $3)$ evidence that the two are causally linked $(1,3,25,31)$.

None of these criteria sets have been completely validated by prospective studies. Also, none of them are able to distinguish mixed causes from pure vascular causes $(5,12)$. 
The gold standard diagnosis for VCI is currently represented by a combination of neuropsychological examinations (eg. Montreal Cognitive Assessment questionnaire) and brain MRI. The presence of MRI abnormalities (hypointensity in T1-weighted image and white matter lesions in T2-FLAIR) can also be used as a predictor of cognitive impairment (11).

VaD clinical characteristics. It is important to emphasize that due to the heterogeneous causes of VCI, there is no typical clinical presentation (16). Patients with VaD usually deteriorate in a step-wise manner, with symptoms becoming more severe with each new stroke. Sometimes, however, dementia can have an abrupt onset as the result of a single stroke (6). The typical neuropsychological profile of VCI include early impairment of attention and executive function, with slowing of motor performance and information processing. Episodic memory is relatively spared compared with that in AD (5). Non-cognitive symptoms in patients with VCI are common and include focal neurologic findings, speech and swallowing disorders, parkinsonism, gait abnormalities and urinary difficulties $(3,12,16)$.

Psychiatric symptoms are as common and important as in AD. Mood symptoms such as depression, emotional lability, and loss of volition (apathy) are especially frequent and persistent in $\mathrm{VaD}$ compared with those in AD (5). The psychiatric symptoms associated with $\mathrm{VaD}$ are divided into those assessed at the patient/carer interview (anxiety, depressed mood, hallucinations and delusions) and symptoms usually found through behavioral observation of the patient (aggression, screaming, restlessness, agitation, wandering, culturally inappropriate behaviors, sexual disinhibition, hoarding, cursing and shadowing). It has been reported that up to $80 \%$ of $\mathrm{VaD}$ patients will manifest them at some point. Usually, these symptoms become more frequent as the severity of dementia increases $(1,33)$.

The history and physical examination should provide information about vascular risk factors, clinical manifestations of cardiovascular disease, hypercoagulable states, migraine, and depression. Also, during the interview it will be established if there are first degree relatives with a history of stroke, vascular disease, or dementia. Physical ex- amination include blood pressure (orthostatic), pulse, body mass index, waist circumference, examination of the cardiovascular system. Neurological exam should note focal neurological signs and possible gait disturbances $(2,25)$.

Neuropsychological tests. In clinical practice it should be used a bedside screening mental status examination that assesses executive function in addition to memory, language and visual-spatial domains. The Neuropsychological Working Group for VCI Harmonization Standards has recommended long, intermediate and short assessment protocols (which take 60,30 or 5 minutes respectively, to administer) along with instruments to evaluate neurobehavioral changes and mood $(4,34)$.

Laboratory tests. Blood tests for evaluation of patients with dementia are useful in excluding co-morbidities and may include the assessment of vitamin B12, folate, thyroid stimulating hormone, homocysteine, calcium, glucose, complete blood cell count, renal and liver function abnormalities. Serological tests for syphilis, Borrelia and HIV should be take into account if there are suggestive clinical features (35). Tests such as electrocardiogram or echocardiogram are useful to assess systemic vascular disease. Also, carotid Doppler images should be performed in all patients with large-vessel events. A lumbar puncture may be recommended in order to differentiate $\mathrm{VaD}$ from normal pressure hydrocephalus (NPH), and also for assessment of the potential contribution of $\mathrm{AD}$ to the clinical picture. It was demonstrated that the profile of tau and amyloid markers in CSF differ between $\mathrm{VaD}$ and $\mathrm{AD}$. Subcortical vascular disease is characterized by normal or slightly increased levels of tau, normal p-tau and decreased levels of Ab42, whereas levels of tau and p-tau are increased in association with decreased levels of Ab42 in AD (1).

Neuroimaging. The neuroimaging tests - MRI or CT scan of the brain - are essential for the diagnosis of $\mathrm{VaD}$. In $\mathrm{VaD}$ patients, $\mathrm{CT}$ is used to rule out causes of cognitive decline other than vascular or neurodegenerative types of dementia, such as a tumor, subdural hematoma, or NPH (1). However, MRI is the most sensitive technique for the diagnosis of $\mathrm{VaD}(1,3)$. Characteristic MR features of $\mathrm{VaD}$ include large-vessel infarcts (arterial territorial and watershed infarcts), signs of small-vessel disease 
(white matter hyperintensities, lacunes and microbleeds) and expressions of neurodegeneration (general atrophy, medial temporal lobe atrophy). Thus, the most important neuroimaging predictors of poststroke dementia are the white matter lesions (WMLs), silent brain infarcts, global cerebral atrophy and medial-temporal lobe atrophy (1). It was shown that stroke patients with more severe WMLs have an increased risk of recurrent strokes and poststroke dementia $(1,29)$. Silent brain infarcts are independent predictors of post-stroke dementia and microbleeds represent radiological signs of CAA. Cortical gray matter atrophy is correlated with the overall severity of cognitive deficit, while the presence of medial - temporal lobe atrophy - a marker of AD- suggests that the post-stroke demetia may occur partially due to a neurodegenerative process that was ongoing in the preclinical phase when the stroke happened $(1,4)$.

\section{DIFFERENTIAL DIAGNOSIS}

A challenge in $\mathrm{VaD}$ diagnosis is to separate the presence of an ongoing neurodegenerative disease (e.g. AD, Parkinsons's disease - PD) from true $\mathrm{VaD}$, considering that the presence of $\mathrm{VaD}$ superimposed on a neurodegenerative process is frequent, especially in older individuals. Both $\mathrm{VaD}$ and NPH involve modifications in the same deep white matter structures and have a similar cognitive profile associated with gait abnormalities and incontinence. In these cases, the final diagnosis will be established by neuroimaging tests. In patients with transcortical sensory aphasia, the diagnosis of dementia is done by reports from the informants, and not by formal cognitive testing because, when is not associated with any other focal neurological sign, this condition can mimic the language deficits often seen in VaD. Studies comparing cognitive impairment in $\mathrm{VaD}$ and $\mathrm{AD}$ have found it difficult to distinguish between the two dementia types. However, some authors suggest that patients with $\mathrm{VaD}$ have greater deficits in frontal-executive functioning and a relative advantage on verbal episodic memory task (1).

$\mathrm{VaD}$ should be differentiated from reversible causes of memory impairment such as: hypothyroidism, anemia, sleep apneas, depression and vitamin deficiencies. The differential diagnosis of $\mathrm{VaD}$ also include other non-degenerative conditions such as: HIV dementia, multiple sclerosis with dementia, progressive multifocal leukoencephalopathy, and head trauma encephalopathy. History usually allows for differentiation of these diseases $(1,9)$.

\section{PROGNOSIS}

VCI does not always progress to dementia, and results from epidemiological and clinical studies shows that improvement in patients with VCI-no dementia is frequent (2).

VCI is assciated with a high risk of post-stroke dementia. Up to a third of people change diagnostic category (no cognitive impairment; cognitive impairment - no dementia; dementia) within 1 year after a stroke. It was shown that, among patients with stroke, those with previous cognitive impairment, polypharmacy, intercurrent stroke and depression are associated with greater rate of cognitive decline $(2,4)$. Compared with $\mathrm{AD}, \mathrm{VCI}$ has a higher rate of mortality and a slower rate of cognitive decline (4).

\section{TREATMENT}

Primary and secondary prevention. VCI may be preventable by vigilant identification and treatment of vascular risk factors. Thus, the treatment of $\mathrm{VaD}$ includes both prevention and symptomatic strategies $(3,4)$. The VCI prevention may be achieved by identifying and treating the cerebrovascular risk factors mentioned above and by lowering stroke risk with antiplatelet or anticoagulant therapy, if indicated $(12,18)$. Non-pharmacological preventive strategies include: participation in stimulating activities (cognitive, physical and social) and dietary intake of antioxidants, B vitamins and omega-3 fatty acids $(1,31)$.

Symptomatic treatment. Recent controlled clinical trials with cholinesterase inhibitors - donepez$\mathrm{il}$, galantamine, and rivastigmine in $\mathrm{VaD}$, as well as galantamine in patients with $\mathrm{AD}$ with $\mathrm{CVD}$, have shown cognitive improvement $(1,3,4)$. The use of the NMDA receptor antagonist, memantine was also associated with symptomatic benefit in $\mathrm{VaD}$ in comparison to placebo $(1,4)$. Currently donepezil is the only agent found to delay the progression from MCI to dementia, although the data were derived from a single study $(9,12,36)$. 
Treatment of behavioral symptoms in VaD. The SSRIs are usually used as a first-line treatment for depression in dementia. Other antidepressants venlafaxine, trazodone and mirtazapine - seems to be well-tolerated alternatives to SSRIs. Among anticonvulsants, carbamazepine can be used in order to attenuate agitation in demented patients. The antipsychotic treatment is usually prescribed when the patient present delusions or hallucinations. The conventional antipsychotics are currently avoided considering their modest efficacy and important side effects (sedation, anticholinergic and extrapyramidal effects). Therefore, currently atypical antipsychotic drugs, risperidone and olanzapine, are considered to be the most effective and also well-tolerated treatment of psychotic symptoms (1).

In all $\mathrm{VaD}$ cases, a proactive, interdisciplinary and holistic approach is strongly recommended in order to improve the patient safety and quality of life (4).

Conflict of interest: none declared Financial support: none declared

\section{REFERENCES}

1. Wahlund L-O, Erkinjuntti T, Gauthier S. Vascular Cognitive Impairment in Clinical Practice. Cambridge University Press 2009. ISBN-13 978-0-521-87537-0.

2. Moorhouse P, Rockwood K. Vascular cognitive impairment: current concepts and clinical developments. Lancet Neurol 2008; 7: 246-55.

3. Godefroy O, Bogousslavsky J. The Behavioral and Cognitive Neurology of Stroke 586-617. Cambridge University Press 2007. ISBN - 13 978-0-521-84261-7.

4. Chui HC, Nielsen-Brown N. Vascular Cognitive Impairment. Continuum Lifeling Learning Neurol 2007; 13(2): 109-143.

5. O'Brien JT, Erkinjuntti T, Reisberg B, Roman G, Sawada T, Pantoni L, Bowler JV, Ballard C, DeCarli C, Gorelick PB, Rockwood K, Burns A, Gauthier S, DeKosky ST. Vascular cognitive impairment. Lancet Neurology 2003; 2:89-98.

6. Health Reference Series Fifth Edition Alzheimer Disease Sourcebook. ISBN 978-0-7808-1150-8

7. Gauthier S, Reisberg B, Zaudig M, Petersen RC, Ritchie K, Broich K, Belleville S, Brodaty H, Bennett D, Chertkow H, Cummings JL, de Leon M, Feldman H, Ganguli M, Hampel H, Scheltens P, Tierney MC, Whitehouse P, Winblad B. Mild cognitive impairment. Lancet 2006; 367: $1262-70$

8. Gauthier S. Clinical Diagnosis and Management of Alzheimer's disease. Third Edition. 2007 Informa UK Limited. ISBN-10: 0415 372992.

9. Levey A, Lah J, Goldstein F, Steenland K, Bliwise D. Mild Cognitive impairment: An Opportunity to Identify Patients at High Risk for Progression to Alzheimer's Disease. Clinical Therapeutics. Vol 28, nr 7, 2006. doi: 10.1016/j.clinthera.2006.07.006 0q 49-2918/06\$19.00.

10. Wang X, Yu Y, Zhao W, LiQ, Li X, Li S, Yin C, Han Y. Altered Whole-Brain Structural Covariance of the Hippocampal Subfields in Subcortical Vascular Mild Cognitive Impairment and Amnestic Mild Cognitive Impairment Patients. Frontiers in Neurology May 2018. Vol. 9 Article 342. doi: 10.3389/fneur.2018.00342.

11. Harris S, Reyhan T, Ramli Y, Prihartono J, Kurniawan JM. Middle Cerebral Artery Pulsatility Index as Predictor of Cognitive Impairment in Hypertensive Patients Frontiers in Neurology July 2018. Vol. 9 Article 538. doi: 10.3389/fneur.2018.00538

12. ACP Medical Knowledge Self-Assessment Program. Neurology. 2015 American College of Physicians. KSAP 17 Neurology. ISBN: 978-1-938245-23-7.

13. Chen CLH, Ikram K, Anqi $Q$, Yin WT, Chen A. The NeuroAiD II (MLC901) in Vascular Cognitive Impariment Study (NEURITES). Cerebrovasc Dis 2013;35(suppl 1):23-29 DOI: 10.1159/000346234

14. Rockwood K, Wentzel C, Hachinski V, HoganDB, MacKnight C, McDowell I: Prevalenceand outcomes of vascular cognitive impairment.Vascular Cognitive ImpairmentInvestigators of the Canadian Study of Healthand Aging. Neurology 2000; 54: 447-451.

15. Wentzel C, Rockwood K, MacKnight C,Hachinski V, Hogan DB, Feldman H, OstbyeT, Wolfson C, Gauthier S, Verreault R, Mc-Dowell I. Progression of impairment in patientswith vascular cognitive impairmentwithout dementia. Neurology 2001; 57: 714-716.

16. Bradley's Neurology in Clinical Practice. Seventh Edition. ISBN: 978-0-323-28783-8.

17. Melzer D, Ely M, Brayne C. Cognitive impairment in elderly people: population based estimate of the future in England, Scotland, and Wales. BMJ 1997; 315: 462.

18. Chen H, Su F, Ye Q, Wang Z, Shu H, Feng Bai F. The Dose-Dependent Effects of Vascular Risk Factors on Dynamic Compensatory Neural Processes in Mild Cognitive Impairment. Frontiers in Aging Neuroscience. Vol. 10 Article 131. May 2018. doi: 10.3389/ fnagi.2018.00131.

19. Barrett-Connor E, Edelstein SL, Corey-Bloom J, Wiederholt WC. Weight loss precedes dementia in community-dwelling older adults. J Am Geriatr Soc 1996;44: 1147-52.

20. Buchman AS, Wilson RS, Bienias JL, Shah RC, Evans DA, Bennett DA. Change in body mass index and risk of incident Alzheimer disease. Neurology 2005; 65(6):892-7.

21. Stewart R, Masaki K, Xue QL et al. A 32-year prospectivestudy of change in body weight and incidentdementia: the Honolulu - Asia Aging Study. ArchNeurol 2005; 62(1): 55-60.

22. Whitmer RA, Gunderson EP, Barrett-Connor E, Quesenberry CP, Jr., Yaffe K. Obesity in middle age andfuture risk of dementia: a 27 year longitudinal populationbased study. Br Med J 2005; 330(7504): 1360.

23. Huadong Z, Juan D, Jingcheng L, Yanjiang W, Meng Z, Hongbo H. Study of the relationship between cigarette smoking, alcohol drinking andcognitive impairment among elderly people in China. Age Ageing (2003)32:205-10. doi: 10.1093/ageing/32.2.205.

24. Richards M, Jarvis MJ, Thompson N,Wadsworth ME. Cigarette smoking andcognitive decline in midlife: evidence from a prospective birth cohort study. Am J Public Health (2003) 93:994-8. doi: 10.2105/ AJPH.93.6.994.

25. Husein M, Jonathan M. Oxford Textbook of Cognitive Neurology and Dementia. Oxford University Press 2016. ISBN 978-0-19-965594-6.

26. DeCarli C. Mild cognitive impairment: prevalence, prognosis, aetiology and treatment. The Lancet Neurology Vol 2 January 2003.

27. Riley KP, Snowdon DA, Markesbery WR. Alzheimer's neurofibrillary pathology and the spectrum of cognitive function: findings from the Nun Study. Ann Neurol 2002; 51: 567-77.

28. Kang Y-C, Zhang L, Su Y,Li Y, Ren W-L, WeiW-S. MicroRNA-26b Regulates the Microglial Inflammatory Response in Hypoxia/ Ischemia and Affects the Development of Vascular Cognitive Impairment. Front. Cell. Neurosci. June 2018. Vol.12 Article 154. https://doi.org/10.3389/fncel.2018.00154. 
29. Leys D, Henon H, Mackowiak-Cordoliani M-A, Pasquier F. Poststroke dementia. Lancet Neurol 2005; 4: 752-9.

30. Linden T, Skoog I, Fagerber B, Steen B, Blomstrand C. Cognitive impairment and dementia 20 months afterstroke. Neuroepidemiology 2004; $23:$ 45-52.

31. Sorbi S, Hort J, Erkinjuntti T, Fladby T, Gainottig G, Gurvith H, Nacmiasa B, Pasquieri F, Popescu B.O, Rektorovak I, Religal D, Rusinan R, Rossoro M, Schmidt R, Stefanovaq E, Warreno JD, Scheltens $\mathrm{P}$ on behalf of the EFNS Scientist Panel on Dementia and Cognitive Neurology. EFNS-ENS Guidelines on the diagnosis and management of disorders associated with dementia. European Journal of Neurology 2012, 19: 1159-1179 doi:10.1111/j.1468-1331.2012.03784.x

32. Tournier-Lasserve E, Joutel A, Melki J et al. Cerebral autosomal dominant arteriopathy with subcortical infarcts and leukoencephalopathy maps on chromosome 19q12. Nat. Genet. 1993. 3, 256-9.
33. Overshott R, Burns A. Treatment of dementia. J Neurol, Neurosurg Psychiatry 2005; 76 (5): 53-9.

34. Hachinski V, ladecola C, Petersen RC et al. National Institute of Neurological Disorders and Stroke - Canadian Stroke Network vascular cognitive impairment harmonization standards. Stroke 2006;37:2220-2241.

35. Filippi M, Agosta F, Barkhof F, Dubois B, Fox NC, Frisoni GB,

36. Jack CR, Johannsen P, Miller BL, Nestor PJ, Scheltens P, Sorbi S, Teipel S, Thompson PM, Wahlund L-O. EFNS task force: the use of neuroimaging in the diagnosis of dementia. European Journal of Neurology 2012, 19: 1487-1511 doi:10.1111/j.1468-1331.2012.03859.

37. Salloway S, Ferris S, Kluger A, et al, for the Donepezil 401 Study Group. Efficacy of donepezil in mild cognitive impairment: A randomized placebo-controlled trial. Neurology. 2004;63:651-657. 